\section{A cross sectional, observational survey to assess levels and predictors of psychological wellbeing in adults with epidermolysis bullosa}

\author{
Emma Dures, ${ }^{1}$ Nichola Rumsey, ${ }^{1}$ \\ Marianne Morris, ${ }^{1}$ Kate Gleeson ${ }^{2}$ \\ 1University of the West of England, \\ Bristol; 2University of Surrey, Guilford, UK
}

\section{Abstract}

Epidermolysis bullosa (EB) describes a cluster of genetically determined skin disorders. Symptoms can be painful, disabling and disfiguring, yet there is little research on the psychological impact of the disease. The study aim was to measure psychological wellbeing in adults with $\mathrm{EB}$; and to examine the association between psychological wellbeing and self efficacy, health locus of control and adjustment to appearance in an observational, cross sectional survey. Questionnaire packs comprising the General Health Questionnaire (GHQ-12), the General Self Efficacy Scale (GSE), the Multidimensional Health Locus of Control Scale (MHLOC), and the Derriford Appearance Scale (DAS-24), were sent to approximately 385 adults with EB. The data were analysed using SPSS. Eighty-seven participants responded. Scores on the GHQ-12 showed non-problematic psychological health in $36 \%$ of the sample; levels bordering on clinical disorder in $32.1 \%$ and severe psychological distress in $31.9 \%$. No correlations were found between demographic factors (age and sex) or clinical factors (EB type and perceived severity) and psychological wellbeing. Scores on the GSE, the internal locus of control sub-scale of the MHLOC and the DAS-24 showed them to be statistically significant correlates of psychological wellbeing $(\mathrm{P}<0.001$; $\mathrm{P}<0.018$; and $\mathrm{P}<0.001$ respectively). In a regression analysis, adjustment to appearance and self efficacy accounted for $24 \%$ of the variation in psychological wellbeing. Adults with EB might be at risk of experiencing poor psychological health. Interventions designed to enhance disease self management, self efficacy and improve body image are likely to be beneficial in this clinical group.

\section{Introduction}

Epidermolysis bullosa (EB) is a lifelong, genetic condition characterised by excessively fragile skin, which blisters with minimal friction. EB is classified into three major types: simplex (EBS); dystrophic (DEB); and junctional (JEB). ${ }^{1}$ It is a very rare condition, although there are limited epidemiological data. The most rigorous estimates from the National EB Registry in the United States suggest that EB affects approximately 8 per one million population, and 19 per one million live births. The National Health Service of the United Kingdom reports that EBS is the most common form (70\%), followed by DEB (25\%), and JEB (5\%) (www.nhs.uk/conditions/Epidermolysis-bullosa). EB blisters are painful open wounds, which can become infected and cause disfigurement and physical disability. ${ }^{2}$ For some, blistering occurs on the hands and feet; for others, it happens throughout the body, including the cornea, stomach, mouth and oesophagus. EB is incurable, and treatment aims to control symptoms by reducing blistering, promoting wound healing, and surgical interventions. ${ }^{3}$

Despite the potentially severe and restrictive nature of EB, a literature review found little research addressing psychological issues. The search was conducted using Ovid, Science Direct and Google Scholar databases, combining the terms: epidermolysis bullosa; skin condition, skin disease; skin disorder; rare condition; chronic condition; psychosocial; psychological; and social impact. Those papers identified focused mainly on affected children and their families. They found a detrimental effect on parents' emotional relationships, employment and leisure time.4,5 From the affected child's perspective, the inability to join in with peers, pain, and disfigurement led to feelings of difference and isolation.6,7 Studies with combined samples of adults and children found that women experienced poorer quality of life than men, and that children were more adversely affected than adults. ${ }^{8}$ Levels of psychological impairment were related to the inability to participate in everyday activities, leading to social isolation and psychological problems. ${ }^{9}$ However, research findings have not consistently highlighted a negative impact on psychosocial health; in a study with 20 hospitalised patients, high levels of psychological wellbeing with adequate, or above adequate, levels of personal development, social integration and affirmation were found. ${ }^{10}$ These contrasting findings might be related to differences in the sampling and study designs (e.g. a community sample responding to a postal survey, compared to a small number of inpatients who provided data in a clinical interview). The recent development of a disease-specific quality of life instrument for use in clinical trials and practice recognises the need for a patient reported outcome measure to generate data from the patient perspective. ${ }^{11}$

Recently, qualitative research with affected adults explored the psychosocial impact of EB. It found that the combination of the disease's rarity, its lifelong and hereditary characteristics,
Correspondence: Emma Dures, University of the West of England, Academic Rheumatology, Bristol Royal Infirmary, Bristol, BS2 8HW, UK.

Tel. +44.117.432 4167.

E-mail: emma2.dures@uwe.ac.uk

Key words: psychological wellbeing, skin condition, disfigurement, self-efficacy, body image.

Contributions: ED, study design, data collection, data analysis; NR, MM, KG, study design, data analysis.

Conflict of interests: the authors declare no potential conflict of interests.

Funding: the study was supported by DEBRA.

Received for publication: 26 October 2012. Revision received: 3 December 2012.

Accepted for publication: 4 December 2012.

This work is licensed under a Creative Commons Attribution NonCommercial 3.0 License (CC BYNC 3.0).

CCopyright E. Dures et al., 2013

Licensee PAGEPress, Italy

Health Psychology Research 2013; 1:e4

doi:10.4081/hpr.2013.e4

and its disfiguring effects were psychologically important; and influenced by participants' ability to contain its impact, and their strategies for managing symptoms and people's reactions to their appearance.12 These findings informed the current study, which mapped the qualitative themes generated with data from adults with $\mathrm{EB}$ onto established psychological variables. The variables selected were self-efficacy and health locus of control, which link to disease containment and symptom management; and body image, which links to the management of disfigurement. Although empirical evidence on the psychosocial impact of EB has been lacking, the need for such research to guide treatment has been acknowledged. 13

Self-efficacy is concerned with an individual's belief in their ability to exercise control and to achieve a desired outcome. It can affect life choices, level of motivation, quality of functioning, resilience to adversity, and vulnerability to stress and depression. ${ }^{14}$ Locus of control refers to an individual's beliefs about whether events in their lives are controlled by internal or external forces. ${ }^{15}$ In a health context, this is defined as perceptions about who is responsible for health outcomes. Control can be classified as internal, external or chance. 16,17 Those with a greater internal locus of control believe that what happens is the consequence of their own actions, and potentially under their control. Those with more of an external locus of control believe events are 
related to something external, and so beyond their control. Those with a chance locus of control believe that their health relies on chance. Internal beliefs have been associated with greater quality of life, and external beliefs with greater levels of depression. ${ }^{18,19}$ The impact of a negative body image on wellbeing in clinical populations is increasingly acknowledged. ${ }^{20}$ Body image is a person's mental picture of their physical self, including perceptions and attitudes to their appearance, attractiveness, health status and sexuality. It incorporates a sense of how their body image influences their interactions, and is therefore a highly significant factor in social and interpersonal relationships. ${ }^{21}$ People with skin conditions may be particularly susceptible to developing a negative body image, exacerbated by appearancerelated stigmatisation. ${ }^{20}$

This study had 2 aims: i) to measure psychological wellbeing in adults with $\mathrm{EB}$; ii) to examine the null hypotheses that there is no association between psychological wellbeing and self-efficacy, an internal health locus of control, and body image.

\section{Materials and Methods}

The design was a cross sectional survey comprising self report measures, plus basic demographic (age and sex) and clinical information (EB type and perceived severity). The outcome measure was the General Health Questionnaire (GHQ-12).22 The GHQ-12 is a measure of current mental health and non-psychotic clinical disorder.23,24 It assesses positive and negative psychological wellbeing, avoids symptoms of physical illness, and is widely used in dermatology populations.25,26 The GHQ-12 has 12 items, which can be scored bimodally (i.e. $-0-0-1-1)$ or as a Likert scale (0-12-3). This study used the Likert scale, with a scoring range of 0 to 36 points; the higher the score, the lower the level of psychological wellbeing. The process measures were the General Self Efficacy Scale (GSE), ${ }^{27}$ the Multidimensional Health Locus of Control Scale (MHLOC) and the Derriford Appearance Scale (DAS-24).28,29 The GSE assesses the belief that one can perform difficult tasks or cope with adversity. GSE scores can range from 10 to 40 points, with higher scores indicating greater self-efficacy. The MHLOC measures expectations in three dimensions of health control: internal, powerful external (doctors and others), and chance external. Form $\mathrm{C}$ was used and made condition-specific by inserting EB into the questions, in accordance with guidelines from the scale's authors. ${ }^{28}$ There are 18 items in total, six relating to each dimension; but there is no total MHLOC score, and all of the subscales are independent of one another. The scores in the three subscales can range from 6 to 36 points; the higher the score, the greater the conviction that this particular concept exerts an impact on one's health. The DAS-24 assesses body image; examining fear, social anxiety, shame, and negative affect. It is designed for use in clinical populations with disfiguring conditions. The DAS-24 comprises 24 questions. Scores can range from 11 to 96 points, with higher scores indicating greater appearance-related distress and dysfunction. The survey also included a rating scale of perceived severity from 1-10, with 1 being very mild, and 10 being very severe.

Ethics approval was granted by the Faculty of Health and Life Sciences Ethics Committee, University of the West of England, Bristol, UK. The survey was piloted with 8 adults with EB, who reported that it was acceptable and feasible to complete. Eligibility criteria were adults with EB, over 18 years old. Recruitment was via 2 healthcare professionals with access to the National EB Register for Scotland and the National EB Register for England and Wales. The sampling frameworks for the two regions differed. The limited size of the Scottish database meant that surveys were posted to all registered adults with EB (115 with EBS and 70 with DEB). The healthcare professional using the register for England and Wales employed a stratified random sampling method and posted 100 surveys to adults with EBS and 100 surveys to adults with DEB. Surveys were sent in March 2008 and data analysis began in May 2008.

\section{Results}

The response rate was approximately $23 \%$ (this approximation is due to incorrect contact details on both databases; 8 packs were returned to the main researcher, plus several to the healthcare professionals). Two responses were excluded because they were completed by an EB-free family member on behalf of an absent relative. Neither of the healthcare professionals who facilitated recruitment identified any patterns in non-respondent character- istics, although the figures showed a higher non-response rate among people with DEB (approximately 17\%, compared to approximately $26 \%$ for EBS). The number of completed and valid surveys was $87(n=87)$.

Statistical analyses were performed using SPSS package for Windows, version 13. The mean (M), standard deviation (SD), Pearson correlation coefficients $(r)$, regression analysis and Student's $t$-test (T) were conducted. Of the 87 participants, 48 were female (55\%) and 39 were male (45\%). Ages ranged from 18 to 90 years (M 48.39, SD 15.31). EB type/subtype was self reported as follows: EBS, 53; DEB, 20; recessive DEB (RDEB), 5; unknown, 7; and did not respond, 2 . The cumulative mortality rate in RDEB is as high as $40 \%$ by age 30 years, making this small sample size unsurprising. ${ }^{30}$ Within ethics guidelines, the healthcare professionals who had helped with recruitment were able to provide useful supplementary data. Taking care not to identify particular individuals, they confirmed that of the seven participants who had responded Not Known; 3 had EBS, and 4 had DEB. The mean score on the perceived severity ratings was 4.23 (M 4.23, SD 2.01). Data were missing for three participants. By EB type, participants with RDEB reported the highest level of severity (M 5.20, SD 2.78), followed by EBS (M 4.52, SD 1.93), DEB (M 3.85 SD 1.95), and EB type unknown (M 2.43, SD 1.27). The sample sizes did not permit meaningful statistical comparisons, but the difference between the mean of participants with RDEB and the mean of participants with EBS was less than one standard deviation and could be attributed to sampling error. RDEB is one of the most clinically severe EB subtypes, while EBS tends to be far milder; suggesting that measures of perceived severity might not equate to measures of clinical severity (Table 1).

The GHQ-12 mean score was 11.73 (SD 4.42); Based on a default threshold of 11-12 for the mean indicating clinical disorder: $36 \%$ of the sample scored $\leq 10 ; 32.1 \%$ scored $11-12$; and $31.9 \%$ scored $\geq 13.31 \mathrm{GSE}$ scores ranged from 24 40 (M 32.57, SD 3.9). MHLOC measures indicated that the majority of the sample believed their health was more dependent on their own behav-

Table 1. Severity rating by Epidermolysis bullosa type.

\begin{tabular}{lllllll}
\hline \multirow{2}{*}{ Epidermolysis bullosa type } & N & Min & Max & Mean & Standard deviation \\
\multirow{2}{*}{ EBS } & Severity & 5 & 1 & 8 & 5.20 & 278 \\
& Valid N (listwise) & 5 & & & & 1.92 \\
\hline \multirow{2}{*}{ DEB } & Severity & 52 & 1 & 8 & 4.52 & 1.95 \\
& Valid N (listwise) & 52 & & & & 3.85 \\
\multirow{2}{*}{ Unknown } & Severity & 20 & 1 & 7 & & 1.27 \\
& Valid N (listwise) & 20 & & & 2.43 & \\
\hline
\end{tabular}

RDEB, recessive dystrophic Epidermolysis bullosa; EBS, simplex Epidermolysis bullosa; DEB, dystrophic Epidermolysis bullosa. 
iours than on chance or other people. The highest mean score was on the internal locus of control sub-scale (M 20.94, SD 7.42), followed by the chance health locus of control sub-scale (M 17.35 , SD 6.33) and the powerful others health locus of control sub-scale (M 12.86, SD 5.48). Scores on the DAS-24 ranged from 48- 68 (M 57.47, SD 4.08) (Table 2).

None of the demographic and clinical variables examined correlated significantly with the outcome measure. The psychological variables, self-efficacy $(\mathrm{P}<0.001)$, internal health locus of control $(\mathrm{P}<0.018)$ and body image $(\mathrm{P}<0.001)$ were significantly correlated (Table 3 ).

A multiple regression analysis provided information on the separate effects and cumulative effects of predictor variables on the outcome measure. The predictor variables were self-efficacy, internal health locus of control, and body image, because the correlation analysis had indicated their statistically significant association with the GHQ-12. Distribution of the residuals was checked and found to be normal (Table 4).

Self-efficacy and body image emerged as predictors, accounting for $24 \%$ of the variation (Multiple $\mathrm{R}=0.519$; adjusted $\mathrm{R}^{2}=0.241$ ), but an internal locus of control was not predictive. Greater self-efficacy was negatively related to poorer psychological wellbeing, while a more negative body image was positively related to poorer psychological wellbeing.

The statistical association of self-efficacy and body image to the demographic variables was examined. No correlation was found between age and self-efficacy. There was a statistically significant negative correlation between age and body image ( $r=-0.250 ; \mathrm{P}=0.022)$, showing a tendency for younger participants to have a more negative body image. An independent samples $t$-test showed that the difference in mean scores for self-efficacy between the male sample and the female sample was not statistically significant. But the difference in mean scores for body image between the male sample and the female sample was statistically significant $(\mathrm{P}=0.059)$, with females having a more negative body image.

\section{Discussion}

This survey examined psychological wellbeing in adults with EB, and the role of self-efficacy, health locus of control, and body image. It was hypothesised that greater self-efficacy, an internal health locus of control, and a more positive body image, would be associated with greater psychological wellbeing. The role of clinical and demographic factors was examined, but there were no hypotheses about their effects because of the mixed findings of previous research.
Scores on the GHQ-12 suggest non-problematic psychological wellbeing in more than one third of the sample, but a substantial proportion with levels bordering on clinical disorder through to severe distress. These means were slightly higher than the mean (M 11.2, SD 5.5) in a general dermatology sample $(n=2579) .32$ GCSE scores were higher than those in a sample of inpatients with psoriasis $(n=100)$, with a mean score of 26.44 (SD 6.69) indicating greater self-efficacy in the EB sample. ${ }^{33}$ In the current study, the highest mean score for MHLOC was on the internal locus of control sub-scale. This contrasts with findings from a study with psoriasis inpatients, which found the highest mean score on the powerful others subscale (M 26.67, SD 5.07).33 The difference could reflect experiences of healthcare. The psoriasis sample involved people in hospital and it might be that powerful others seemed more important in that context. In contrast, data from a community sample of adults with EB indicated that many self-manage their disease. ${ }^{12}$ Scores on the DAS 24 were slightly higher than the mean (M 30.99, SD 13.88) for the general population ( $n=1107)$, and considerably lower than the mean (M 47.7, SD 17.8) in a clinical population comprising adult patients awaiting reconstructive plastic surgery for an objectively identifiable abnormality of appearance $(n=535) .{ }^{29}$ The standard deviation for all these samples is large, suggesting considerable within-group variation. Overall, scores indicate that body image did not seem particularly negative in the present study sample.

No correlations were found between demo-

Table 2. Scores on the measures assessing psychological wellbeing, health locus of control, self efficacy and adjustment to appearance.

\begin{tabular}{lccccc} 
General Health & N & Min & Max & Mean & $\begin{array}{c}\text { Standard } \\
\text { deviation }\end{array}$ \\
Questionnaire 12 total & 86 & 4 & 27 & 11.73 & 4.42 \\
Self efficacy scale total & 84 & 24 & 40 & 32.57 & 3.90 \\
\hline Internal health locus of control total & 84 & 6 & 36 & 20.94 & 7.42 \\
Chance health locus of control total & 85 & 6 & 36 & 17.35 & 6.33 \\
\hline Powerful health locus of control total & 84 & 6 & 34 & 12.86 & 5.48 \\
Total & 86 & 48 & 68 & 57.47 & 4.08 \\
Valid N (listwise) & 83 & & & & \\
\hline
\end{tabular}

Table 3. Correlation coefficients ( $r$ ) between General Health Questionnaire (GHQ-12) and the examined predictor variables.

\begin{tabular}{lc} 
Examined variables & $r$ \\
Age & -0.018 \\
Severity & 0.152 \\
\hline EB type & -0.065 \\
Sex & -0.125 \\
\hline Generalised self efficacy & $-0.370^{* *}$ \\
Internal health locus of control & $-0.257^{*}$ \\
\hline External chance health locus of control & 0.064 \\
External powerful health locus of control & 0.110 \\
\hline Adjustment to appearance & $0.405^{* *}$
\end{tabular}

${ }^{*} \mathrm{P}<0.005,{ }^{* *} \mathrm{P}<0.001$

Table 4. Predictors of General Health Questionnaire (GHQ-12) in adults with epidermolysis bullosa.

\begin{tabular}{lccccc}
\hline Psychological wellbeing predictors & B & SEB & Beta & T & Sig T \\
Self efficacy & -0.307 & 0.115 & -0.269 & -2.680 & 0.009 \\
Internal health locus of control & -0.104 & 0.059 & -0.173 & -1.769 & 0.081 \\
\hline Adjustment to appearance & 0.104 & 0.034 & 0.307 & 3.058 & 0.003 \\
\hline
\end{tabular}

B, unstandardised coefficient; SEB, standard error of B; Beta, standardised coefficient; T, $t$-test value; Sig T, significance (T). 
graphic factors (age and sex), or clinical factors (EB type) and psychological wellbeing. Although inferences about the role of clinical variables should be drawn with caution as the diagnostic categories were self reported and some sample sizes were small, the evidence does not support a straightforward relationship between EB type and psychological impact. This supports research findings on children with EB, which highlight some variation in psychological impact between types, but conclude that differences are not clearly defined, extensive or predictable on that basis.6,7 The psychological variables examined (self-efficacy, health locus of control, and body image) were statistically significant correlates of psychological wellbeing. In a regression analysis, an internal health locus of control was not a statistically significant predictor of psychological wellbeing, although this may have been an effect of the small sample size. Body image was the strongest predictor, followed by self-efficacy. Together they accounted for $24 \%$ of the variance in the outcome measure. The results suggest that a stronger belief in one's ability to gain control and a more positive body image contribute to better psychological wellbeing. However, future research should explore other potential predictors, such as pain, disability and social support.

These findings have several implications for support provision. Self-efficacy is well established as a common pathway through which psychosocial factors affect health functioning and outcomes, and is the organising focus of many self-management programmes. ${ }^{34,35}$ When designing interventions, it is important to understand the specific concerns of the target group. ${ }^{36}$ Future research should examine the potential of EB-specific versus generic selfmanagement programmes, including the modes of delivery. This could be particularly relevant for a condition as rare as EB; as few healthcare professionals have experience of the clinical and psychosocial complexity of the condition, and those affected are geographically dispersed. 37 This current study found that an internal health locus of control correlated with psychological wellbeing, but was not a statistically significant predictor. Although self efficacy and health locus of control are related, there is a distinction. Health locus of control refers to an expectation about whether one's health is controlled by one's own behaviour or external forces. Self efficacy focuses on beliefs about one's capacity to undertake behaviour. Individuals might believe they are personally responsible for their health, but if they lack the skills or information needed to adopt the appropriate health behaviour, they would experience a sense of futility. 38 This difference is potentially significant for designing interventions, as self-management requires knowledge and resources in addition to people's sense of their role in managing their condition. ${ }^{39}$ The impact of disfigurement is increasingly acknowledged in the dermatology literature. For example, Langley, Krueger and Griffiths (2005) found that the two main contributors to stress in people with psoriasis were engaging in avoidance behaviours, and the belief that they were being evaluated on the basis of their skin condition. ${ }^{40}$ Although limited, there is evidence that interventions based on a cognitive behavioural approach can effectively address body image concerns, and associated social anxiety and avoidance. For example, significant reductions in anxiety, depression and appearance concerns have been found with an online intervention for individuals with visible differences. 41

\section{Limitations}

The low response rate (23\%) raised concerns about non-response bias. This rate is not uncommon for postal surveys, with the groups less likely to respond including younger adults, those with poorer health and greater disabilities, a low income, and a low level of education. ${ }^{42}$ This might account for the lower response rate among people with DEB, as those affected can experience high levels of disability. The length and the sensitivity of the survey might have affected the response rate. Research for the EB register in England and Wales found that high numbers of people with EB missed schooling as a result of their condition; this impact on education has also emerged in qualitative studies. ${ }^{12}$ In addition, Moss (2008) identified the potential for people with EB to experience secrecy and shame, which could contribute to a decision not to respond. 43 The above factors suggest that finding the survey demanding to complete and having negative feelings about their skin condition and appearance might have influenced the decision to participate. Finally, relying on last known addresses is often an issue in postal studies. ${ }^{44}$ Despite the response rate, the sample size for a small, hard-to-reach population is acceptable, particularly in exploratory research. ${ }^{45}$ Although generalisations cannot be made with confidence, the findings allow for cautious suggestions about the psychological impact of EB.

\section{Conclusions}

Greater self-efficacy and a more positive body image could contribute to higher levels of psychological wellbeing in adults with EB. Interventions that increase belief in the ability to regain control, and enhance body image and social confidence could be beneficial in this clinical group. This proposal is made cautiously, given the low response rate and the percent- age of variance in psychological wellbeing not accounted for; and further research is recommended into other potentially influential variables. These findings contribute to understanding the psychological impact of a rare condition in an under-researched area.

\section{References}

1. Mallipeddi R. Epidermolysis bullosa and cancer. Clin Exp Dermatol 2002;27:616-23.

2. Azizkhan R, Denyer J, Mellerio J, et al. Surgical management of epidermolysis bullosa: Proceedings of the IInd international symposium on epidermolysis bullosa, Santiago, Chile, 2005. Int J Dermatol 2007;46:801-8.

3. Gannon B. Epidermolysis bullosa: pathophysiology and nursing care. Neonat Netw 2004;23:25-32.

4. Fine J, Johnson L, Weiner M, et al. Impact of inherited epidermolysis bullosa on parental interpersonal relationships, marital status and family size. Brit J Dermatol 2005;152:1009-14.

5. Van Scheppingen C, Lettinga A, Duipmans $\mathrm{J}$, et al. The main problems of parents of a child with epidermolysis bullosa. Qual Health Res 2008;18:545-56.

6. Van Scheppingen C, Lettinga A, Duipmans $\mathrm{J}$, et al. Main problems experienced by children with epidermolysis bullosa: a qualitative study with semi-structured interviews. Acta Derm Venereol 2008;88: 143-50.

7. Williams E, Gannon K, Soon K. The experiences of young people with epidermolysis bullosa simplex: A qualitative study. J Health Psychol 2011;16:701-10.

8. Tabolli S, Sampogna F, Di Pietro C, et al. Quality of life in patients with epidermolysis bullosa. Brit J Dermatol 2009;161:86977.

9. Horn H, Tidman M. Quality of life in epidermolysis bullosa. Clin Exp Dermatol 2002;27:707-10.

10. Andreoli E, Mozzetta A, Angelo C, et al. Epidermolysis bullosa: psychological and psychosocial aspects. Dermatol Psychosom 2002;3:77-81.

11. Frew J, Murrell D. Quality of life measurements in epidermolysis bullosa: tools for clinical research and patient care Dermatol Clin 2010;28:185-90.

12. Dures E, Morris M, Gleeson K, Rumsey N. The psychosocial impact of epidermolysis bullosa. Qual Health Res 2011;21:771-82.

13. Pagliarello C, Tabolli S. Factors affecting quality of life in epidermolysis bullosa Expert Rev Pharmacoecon Outcomes Res 2010;10:329-38.

14. Bandura A. Health promotion by social 
cognitive means. Health Educ Behav 2004;31:143-64.

15. Rotter J. The development and applications of social learning theory 1982 . New York: Praeger; 1982.

16. Wallston $\mathrm{K}$, Wallston $\mathrm{B}$, DeVellis $\mathrm{R}$. Development of the multidimensional health locus of control (MHLC) scales. Health Educ Monographs 1978;6:160-70.

17. Laffrey S, Isenberg $M$. The relationship of internal locus of control, value placed on health, perceived importance of exercise, and participation in physical activity during leisure. Int J Nurs Stud 2003;40:453-9.

18. Pimm T, Weinman J. Applying Leventhal's self regulation model to adaptation and intervention in rheumatic disease. Clin Psychol Psychot 1998;5:62-75.

19. Kiviruusu 0, Huurre T, Aro H. Psychosocial resources and depression among chronically ill young adults: Are males more vulnerable? Soc Sci Med 2007;65:173-86.

20. Wenninger K, Weiss C, Wahn U, Staab D. Body image in cystic fibrosis-development of a brief diagnostic scale. J Behav Med 2003;26:81-94.

21. Luskin Biordi D, Warner A, Knapik G. Body image. In: Morof Lubkin I, Larsen P, eds. Chronic illness: impact and interventions 6th edn. Sudbury: Jones \& Bartlett, 2006. pp 181-197.

22. Belhadjali H, Amri M, Mecheri A, et al. Vitiligo and quality of life: a case-control study. [Vitiligo et qualite de vie: etude castemoins] Ann Dermatol Venereol 2007; 134:233-6.

23. Goldberg D. Manual of the General Health Questionnaire. Windsor: NFER Publishing; 1978.

24. Tait R, French D, Hulse G. Validity and psychometric properties of the General Health Questionnaire-12 in young Australian adolescents. Aust NZ J Psychiat 2003;37:37481.

25. Hu Y, Stewart-Brown S, Twigg L, Weich S. Can the 12-item general health questionnaire be used to measure positive mental health? Psychol Med 2007;37;1005-13.

26. Mease P, Menter M. Quality-of-life issues in psoriasis and psoriatic arthritis: Outcome measures and therapies from a dermatological perspective. J Am Acad Dermatol 2006;54:685-704.

27. Schwarzer R, Jerusalem M. Generalized self-efficacy scale. In: Weinman J, Wright S, Johnston M eds. Measures in health psychology: a user's portfolio. Causal and control beliefs. Windsor: NFER-NELSON; 1995. pp 35-37.

28. Wallston K, Stein M, Smith C. Form C of the MHLC scales: a condition-specific measure of locus of control. J Pers Assess 1994;63:534-53.

29. Carr T, Moss T, Harris D. The DAS24: a short form of the Derriford appearance scale DAS59 to measure individual responses to living with problems of appearance. Brit J Health Psychol 2005;10: 285-98.

30. Farhi D. Surgical management of epidermolysis bullosa: the importance of a multidisciplinary management. Int J Dermatol 2007;46:815-6.

31. Goldberg D, Gater R, Sartorius N, et al. The validity of two versions of the GHQ in the WHO study of mental illness in general health care. Psychol Med 1997;27:191-7.

32. Picardi A, Abeni D, Pasquini P. Assessing psychological distress in patients with skin diseases: Reliability, validity and factor structure of the GHQ-12. J Eur Acad Dermatol 2001;15:410-7.

33. Zalewska A, Miniszewska J, Chodkiewicz J, Narbutt J. Acceptance of chronic illness in psoriasis vulgaris patients. J Eur Acad Dermatol Venereol 2007;21:235-42.

34. Bodenheimer T, Lorig $\mathrm{K}$, Holman $\mathrm{H}$, Grumback K. Patient self-management of chronic disease in primary care. J Amer Med Assoc 2002;288:2469-75.

35. Marks R, Allengrante J, Lorig K. A review and synthesis of research evidence for self-efficacy-enhancing interventions for reducing chronic disability: implications for health education practice (part 1). Health Promot Pract 2005;6:37-43.

36. Lorig K, Holman H. Self-management education: history, definition, outcomes, and mechanisms. Ann Behav Med 2003;26:1-7.

37. Dures E, Morris M, Gleeson K, Rumsey N. You're whatever the patient needs at the time. The impact on health and social care professionals of supporting people with epidermolysis bullosa. Chronic Illn 2010;6: 215-27.

38. Mabe Newman A. Self efficay. In: Lubkin I, Larsen P eds. Chronic illness: impact and interventions 2006 (6th Edition). Canada: Jones and Bartlett; 2006.

39. De Silva D. Helping people help themselves: a review of the evidence considering whether it is worthwhile to support self-management. The Health Foundation 2011: London.

40. Langley R, Krueger G, Griffiths C. Psoriasis: epidemiology, clinical features, and quality of life. Ann Rheum Dis 2005; 64:18-25.

41. Bessell A, Brough V, Clarke A, et al. Evaluation of the effectiveness of face it, a computer-based psychosocial intervention for disfigurement-related distress. Psychol Health Med 2012;17:565-77.

42. Sheldon H, Rasul F. Increasing response rates among BME and other hard to reach groups: a review of literature relevant to the national acute patients' survey. Picker Institute: making patients' views count 2006. Available from: www.pickereurope.org

43. Moss K. Contact at the borderline: psychoanalytical psychotherapy with EB patients Brit J Nurs 2008;10-23;17:449-55.

44. Edwards P, Roberts I, Clarke M, et al. Increasing response rates to postal questionnaires: systematic review. BMJ 2002; 324:1183.

45. Crosby R, Salazar L, Di Clemente R, Lang D. Balancing rigor against the inherent limitations of investigating hard-to-reach populations Health Educ Res 2010;25:1-5. 